\title{
Gait control: a specific subdomain of executive function?
}

\author{
Olivier Beauchet ${ }^{1,2,3^{*}}$, Cédric Annweiler ${ }^{1,2,3,4}$, Manuel Montero-Odasso ${ }^{4}$, Bruno Fantino ${ }^{1,2,3}$, François R Herrmann ${ }^{5}$ \\ and Gilles Allali ${ }^{6}$
}

\begin{abstract}
Background: Few studies looked at the association between gait variability and executive subdomains (ESD). The aim of this study was to examine the association between ESD (i.e., information updating and monitoring) and stride time variability among healthy older adults.

Methods: Seventy-eight healthy older adults (mean age $69.9 \pm 0.9$ years, 59\% women) were divided into 3 groups according to stride time variability (STV) tertiles while steady state walking. Coefficient of variation of stride time was used as a marker of STV. Scores on cognitive tests evaluating information updating and monitoring (Digit Span test), mental shifting (Trail Making Test part A and part B) and cognitive inhibition (Stroop Color Word test) were used as measures of ESD.
\end{abstract}

Results: The full adjusted and the stepwise backward logistic regression models showed that the highest tertile (i.e., the worst performance) of STV was only associated with lower Digit Span performance (Odds ratio $=0.78$ with $\mathrm{P}=0.020$ and Odds ratio $=0.81$ with $\mathrm{P}=0.019$ ).

Conclusions: Information updating and monitoring are associated with STV in the sample of studied participants, suggesting that walking may be a complex motor task depending specifically of this subdomain of executive functions.

Keywords: Gait disorders, Cognition, Motor impairment, Normal aging, Executive functions, Aging research

\section{Background}

Walking is considered as an automatic motor activity mainly controled by subcortical and spinal regions [1]. Stride-to-stride variability of stride time (STV) is a measure of the reliability of lower limb movements and is a dependable marker of the rhythmic stepping mechanism [2,3]. A low STV (i.e.; under 5\%) reflects an efficient gait control [3]. The past decade highlighted that cognitive functions, and in particular executive functions $(\mathrm{EF})$, are associated with STV and play an important role in gait control even in routine walking condition [4-7]. EF are heterogeneous cognitive functions [8]. Based on Miyake's model, executive functions-mental set may be divided in 3 main separate executive subdomains (ESD), which are information updating and monitoring, mental shifting and cognitive inhibition [8]. Little is known

\footnotetext{
* Correspondence: olbeauchet@chu-angers.fr

'UPRES EA 2646, University of Angers, UNAM, France

Full list of author information is available at the end of the article
}

about the association between these ESD and STV among healthy participants [7]. Safe gait requires to analyse an important flow of visual, vestibular and proprioceptive information, and to update these information in a short period of time, in order to adapt gait to the environmental conditions [3,5]. Thus, we hypothesized that STV could be associated with a specific ESD which is information updating and monitoring. The aim of this study was to examine whether there was an association between information updating and monitoring and STV among healthy older adults.

\section{Methods}

Participants

Between November 2009 and February 2011, 78 healthy older adults (mean age $69.9 \pm 0.9$ years, $59 \%$ women) were recruited from the GAIT (Gait and Alzheimer Interaction Tracking) study which is a cross-sectional study designed to compare gait characteristics of $\mathrm{AD}$
C Biomed Central

() 2012 Beauchet et al; licensee BioMed Central Ltd. This is an Open Access article distributed under the terms of the Creative Commons Attribution License (http://creativecommons.org/licenses/by/2.0), which permits unrestricted use, distribution, and reproduction in any medium, provided the original work is properly cited. 
patients with healthy participants. In the present data, we focused on the gait characteristics of healthy participants.

\section{Clinical assessment}

The number of drugs taken daily, height $(\mathrm{m})$ and weight $(\mathrm{kg})$ were recorded. Body mass index (BMI) was calculated as weight $/$ height $t^{2}\left(\mathrm{~kg} / \mathrm{m}^{2}\right)$. The participants were interviewed with standardized questionnaires gathering information about history of falls over the past year. A fall was defined as an event resulting in a person coming to rest unintentionally on the ground or at other lower level, not as the result of a major intrinsic event or an overwhelming hazard. Education level was evaluated with the number of years at school. The highest level of education corresponded to the highest number of years at school. ESD were evaluated based on Miyake's model that distinguishes mental shifting from information updating and cognitive inhibition [8]. We used the direct (i.e.; forward) and indirect (i.e.; backward) Digit Spans test for the evaluation of information updating and monitoring [9], the Trail Making Test (TMT) part A and part B for the evaluation of the mental shifting constituent [10], and the Stroop Color Word test for the evaluation of cognitive inhibition [11]. Digit spans test examines the ability to recall a sequence of numbers forward and backward in corrected order immediately after its presentation. It is a common measure of working memory that keeps several pieces of information active while we try to do something with them during a short period of time under 3 minutes. This management of information is based on monitoring and updating processes. The TMT evaluated two specific aspects of EF which are the visual attention and the task switching. This test requires a subject to "connect-the-dots" of 25 consecutive targets on a sheet of paper or a computer screen. Two versions are available: part $\mathrm{A}$ in which the targets are all numbers $(1,2,3$, etc.), and part $B$ in which the subject alternates between numbers and letters (1, A, 2, B, etc.). The goal is to perform the test as quickly as possible, and the time taken to complete it is used as the primary performance metric. The Stroop color test is considered to specifically measure selective attention and cognitive inhibition. It is composed of three boards: board 1 shows color names written in black ink; board 2 shows color rectangles; board 3 shows color names written in color ink. Board after board, the subject must either read, or name the colors as quickly as possible, from right to left going to the following line at each end of the line. The number of words which has been read or color names found within 45 seconds is measured. The test is performed in the following order, with the following instructions: color naming (board showing color rectangles), reading of color names (board showing color names written in black) and interference situation (board showing color names written in color). For each board, the experimenter counts the number of words that have been read or colors named within the 45 seconds. The test-retest reliability of all these cognitive tests was moderate to high $[12,13]$. The total number of direct and indirect digit recalls in correct order, a ratio score of TMT (i.e., TMTB/TMTA) and a ratio score of Stroop (i.e., ("No Interference" [color])/ "Interference" [color - word]) were used as outcomes.

\section{Gait recordings}

STV was measured using SMTEC ${ }^{\circledR}$ system $\left(\right.$ SMTEC $^{\circledR}$, Sport \& Medical Technologies SA, Nyon Switzerland) [14]. The participants were asked to walk at their usual self-selected walking speed on a 10-meter walkway in a 20 -meter corridor. According to the guidelines for spatiotemporal gait analysis in older adults and to assure that gait parameters were collected while steady state walking, participants started walking at least 2 meters before reaching the 10-meter walkway and completed their walk at least two meters beyond it [15]. Participants walked one trial in a quiet, well-lit corridor wearing their own footwear. Coefficient of variation $(\mathrm{CoV})(\mathrm{CoV}=$ (standard deviation $/$ mean $) \times 100$ ) of stride time was used as a marker of STV. Little is known about the test-retest reliability of STV but it has been recently reported that the immediate reliability of the $\mathrm{CoV}$ of stride time while single and dual tasking is poor [16].

\section{Statistics}

The participants' baseline characteristics were summarized using means and standard deviations or frequencies and percentages, as appropriate. For the current analysis, participants were separated into 3 groups based on the tertilization of STV: lowest tertile (mean STV of $1.2 \pm$ $0.3 \%$; range [0.6-1.5]; $\mathrm{n}=26$ ), intermediate tertile (mean value of $1.8 \pm 0.1 \%$; range [1.6-2.0]; $\mathrm{n}=26$ ) and highest tertile (mean value of $2.9 \pm 1.1 \%$; range [2.1-6.4]; $\mathrm{n}=26$ ). Comparisons among groups were performed using oneway analysis of variance (ANOVA) with Bonferroni corrections or Chi-square test, as appropriate. Multiple logistic regression analyses were performed to specify the association between the highest STV tertile (dependent variable) and the ESD which was different across the 3 groups of participants (independent variable) adjusted for participants' baseline characteristics (age, gender, BMI, number of drugs taken per day, falls during the past year and education level). P-values less than 0.05 were considered as statistically significant. All statistics were performed using SPSS (version 17.0; SPSS, Inc., Chicago, IL).

\section{Standard Protocol Approvals, Registrations, and Patient Consents}

Participants in the study were included after having given their written informed consent for research. The 
study was conducted in accordance with the ethical standards set forth in the Helsinki Declaration (1983). The entire study protocol was approved by the local Ethical Committee of Angers (France).

\section{Results}

Table 1 showed the clinical characteristics of the studied sample of participants according to the tertiles of $\mathrm{CoV}$ of STV while walking alone in the studied population. Based on the established cut-off points from STV tertilization, gender $(P=0.003)$ and history of falls $(P=0.014)$ differed between groups, participants in the lowest tertile being less frequently female than those in the intermediate tertile $(P=0.001)$, and less frequently fallers than those in the intermediate $(\mathrm{P}=0.004)$ and highest tertiles $(\mathrm{P}=0.017)$. According to ESD assessment, only performance on Digit Span test differed between groups ( $\mathrm{P}=$ 0.004), those in the lowest tertile of STV (i.e., the best gait performance) having enumerated more figures than those in the highest tertile $(P=0.004)$. There was no significant difference for the others characteristics.

As shown in Table 2 the full adjusted and the stepwise backward logistic regression models showed that the highest STV tertile was associated with lower performance on Digit Span (Odds ratio [OR] $=0.78$ with $\mathrm{P}=0.020$ and $\mathrm{OR}=0.81$ with $\mathrm{P}=0.019$, respectively). There was no association between STV and the other covariates.

\section{Discussion}

Our results show that gait control assessed by the STV was associated with a specific ESD, the information updating and monitoring assessed with the Digit Span test. More precisely, an increase of one unit in the variability of stride time was significantly associated with a $1.28(1 / 0.78)$ fold decrease one unit in digit span score, which corresponds to a $28 \%$ decrease.

Previous studies have already shown an independent association between EF performance and gait $[4-7,17]$. Nevertheless, most of them focused on participants with neurodegenerative diseases such as patients with the behavioural variant of the frontotemporal degeneration [6]. Here, we showed that gait of healthy older adults is a motor task requiring the involvement of higher-level cognitive input even in steady-state while usual walking. Few studies have explored the association of EF with gait performance among healthy older adults [4,5,17]. Some of them found that a low performance on global EF was associated with a low gait speed and its rate of gait speed decline, but none found that the impairment in a specific ESD could account for slowing of usual gait $[4,17]$. Difference with our findings could be due to the use of a different gait characteristic. STV compared to gait speed is indeed a specific temporal gait parameter which has been related to gait control [3]. Furthermore, in a previous study comparing the relationship between gait control

Table 1 Clinical characteristics of the studied sample of participants according to the tertiles of stride-to-stride variability of stride time (STV) while walking alone $(n=78)$

\begin{tabular}{|c|c|c|c|c|c|c|c|}
\hline & \multicolumn{3}{|c|}{ Tertiles of STV } & \multicolumn{4}{|c|}{ P-Value* } \\
\hline & $\begin{array}{l}\text { Lowest } \\
(n=26)\end{array}$ & $\begin{array}{l}\text { Intermediate } \\
(n=26)\end{array}$ & $\begin{array}{l}\text { Highest } \\
(n=26)\end{array}$ & Overall & $\begin{array}{c}\text { Lowest } \\
\text { versus intermediate }\end{array}$ & $\begin{array}{c}\text { Lowest } \\
\text { versus highest }\end{array}$ & $\begin{array}{c}\text { Intermediate } \\
\text { versus highest }\end{array}$ \\
\hline Age (years), mean \pm SD & $70.0 \pm 1.0$ & $69.7 \pm 0.7$ & $69.8 \pm 0.8$ & 0.510 & - & - & - \\
\hline Female, n (\%) & $9(34.6)$ & $21(80.8)$ & $16(61.5)$ & 0.003 & 0.001 & 0.052 & 0.126 \\
\hline $\mathrm{BMI}\left(\mathrm{kg} / \mathrm{m}^{2}\right)$, mean $\pm \mathrm{SD}$ & $24.8 \pm 2.6$ & $25.6 \pm 3.3$ & $26.6 \pm 4.4$ & 0.187 & - & - & - \\
\hline Number of drugs taken daily, mean \pm SD & $2.0 \pm 1.5$ & $2.5 \pm 2.0$ & $2.7 \pm 2.5$ & 0.406 & - & - & - \\
\hline Falls during the past year, n (\%) & $2(7.7)$ & $11(42.3)$ & $9(34.6)$ & 0.014 & 0.004 & 0.017 & 0.569 \\
\hline Education levelt (years), mean \pm SD & $11.8 \pm 3.3$ & $10.9 \pm 3.2$ & $11.8 \pm 3.7$ & 0.550 & - & - & - \\
\hline \multicolumn{8}{|l|}{ Executive subdomain performance } \\
\hline Digit Span scoref, mean \pm SD & $15.5 \pm 3.6$ & $13.5 \pm 3.2$ & $12.7 \pm 2.3$ & 0.004 & 0.060 & 0.004 & 1.000 \\
\hline Ratio score TMTB/TMTA\#, mean \pm SD & $2.1 \pm 0.6$ & $2.2 \pm 0.6$ & $2.0 \pm 0.5$ & 0.486 & - & - & - \\
\hline $\begin{array}{l}\text { Ratio score Stroop Part III/ Part I, } \\
\text { mean } \pm \text { SD }\end{array}$ & $2.9 \pm 0.8$ & $3.2 \pm 0.8$ & $3.2 \pm 1.6$ & 0.530 & - & - & - \\
\hline
\end{tabular}

BMI: Body mass index

TMTA: Trail Making Test A

TMTB: Trail Making Test B

* Comparison based on oneway ANOVA, Kruskal-Wallis test with Bonferroni corrections or Chi-square test, as appropriate

$t$ : Assessed with the number of years at school

\#: Total number of digits that a subject can absorb and recall in correct forward and backward serial orders after hearing them

\#: Times to "connect-the-dots" as quickly as possible of 25 consecutive targets on a sheet of paper; part A the targets are numbers, and part B alternated numbers and letters

: Ratio score Stroop Color Word test Part III/ Part I (Part I corresponding to time to name color, and part III corresponding to time to name the color of incongruent color words)

P significant $(<0.05)$ indicated in bold. 
Table 2 Multiple logistic regression models showing the association between the highest tertile of stride-to-stride variability of stride time (dependent variable) and Digit Span score (independent variable) adjusted for clinical characteristics $(\mathbf{n}=\mathbf{7 8})$

\begin{tabular}{|c|c|c|c|c|c|c|}
\hline & \multicolumn{3}{|c|}{ Full adjusted model } & \multicolumn{3}{|c|}{ Stepwise backward model } \\
\hline & OR & $95 \% \mathrm{Cl}$ & P-value & OR & $95 \% \mathrm{Cl}$ & P-value \\
\hline Digit Span score * & 0.78 & {$[0.63 ; 0.96]$} & 0.020 & 0.81 & {$[0.68 ; 0.97]$} & 0.019 \\
\hline Age & 1.02 & {$[0.50 ; 2.10]$} & 0.954 & - & - & - \\
\hline Female & 1.05 & {$[0.32 ; 3.4]$} & 0.932 & - & - & - \\
\hline BMI & 1.09 & {$[0.94 ; 1.27]$} & 0.253 & - & - & - \\
\hline Education level † & 1.11 & {$[0.95 ; 1.30]$} & 0.182 & - & - & - \\
\hline Number of drugs taken daily & 1.00 & {$[0.77 ; 1.30]$} & 0.984 & - & - & - \\
\hline History of falls $\neq$ & 2.07 & {$[0.57 ; 7.54]$} & 0.270 & - & - & - \\
\hline
\end{tabular}

OR: Odds ratio

$\mathrm{Cl}$ : confident interval

BMI: body mass index

*: Total number of digits that a subject can absorb and recall in correct forward and backward serial orders after hearing them

$t$ : Assessed with the number of years at school

\#: Falls in the previous year

Odds ratio significant (i.e.; $<0.05$ ) indicated in bold.

and higher-level cognitive resources, Hausdorff et al. also showed that STV was specifically associated with EF, but not with memory or global cognitive functions [5]. The involvement of EF we observed among healthy older adults could be explained by age-related changes in sensorimotor system leading to a decrement in automaticity compensated by an increased involvement of EF to properly process all sensorimotor information. Recently, this hypothesis was reinforced by a MRI study which showed that white matter hyperintensity load correlates with EF dysfunctions as well as gait and posture measures [18]. The association between STV and updating and monitoring function shown in our study is not surprising because walking requires processing a flow of information in a short period to adapt gait to the environmental conditions. These results open new perspectives in the improvement of fall prediction and of gait rehabilitation. Indeed, because dementia-related gait disorders have been associated to central misprocessing of information due to EF dysfunctions, it could be suggested that impairment of updating and monitoring shown in demented older adults may be used as a predictor of falls. Furthermore, gait rehabilitation based on an improvement of updating and monitoring function could be a new way to prevent falls.

The main limitation of our study was the relatively small number of participants included in the current analysis. In addition, although we were able to control for many characteristics, residual potential confounders may still remain.

\section{Conclusions}

Taken together, previous and our findings provide a new insight into gait control. Even relatively simple, steady- state walking should be considered more as a complex motor task than an automated and rhythmic motor task. Based on Miyake's model, our results highlighted that gait control is associated with one specific ESD, the information updating and monitoring. Future investigations should investigate whether this association is also observed among younger participants or is a consequence of aging.

\section{Acknowledgements}

Gilles Allali was supported by a grant from the Swiss National Science Foundation (No 33CM30-124115). The study was supported by the French Ministry of Health (Projet Hospitalier de Recherche Clinique national n²009A00533-54) and the "Gérontopôle des Pays de la Loire". The sponsor had no role in the design and conduct of the study, in the collection, management, analysis, and interpretation of the data, or in the preparation, review, or approval of the manuscript.

\section{Author details}

${ }^{1}$ UPRES EA 2646, University of Angers, UNAM, France. ${ }^{2}$ Department of Neuroscience, Division of Geriatric Medicine, Angers University Hospital, Angers, France. ${ }^{3}$ Angers University Memory Clinic, Angers, France. ${ }^{4}$ Department of Medicine, Division of Geriatric Medicine, the University of Western Ontario, London, Ontario, Canada. ${ }^{5}$ Department of Internal Medicine, Rehabilitation and Geriatrics, Geneva University Hospitals and University of Geneva, Geneva, Switzerland. 'Department of Neurology, Geneva University Hospital and University of Geneva, Geneva, Switzerland.

\section{Authors' contributions}

$\mathrm{OB}$ has full access to the data in the study and takes responsibility for the integrity of the data and the accuracy of the data analyses. Study concept and design: GA and $\mathrm{OB}$. Acquisition of data: $\mathrm{CA}$ and BF. Analysis and interpretation of data: $O B, G A, F R H, V D, R W K$ and $C A$. Drafting of the manuscript: $O B, C A$ and $G A$. Critical revision of the manuscript for important intellectual content: $M M O, F R H$ and BF. Statistical expertise: FRH. Administrative, technical, or material support: CA. Study supervision: OB and GA. All authors read and approved the final manuscript.

\section{Competing interests}

The authors declare that they have no competing interests. There were not any financial and personal relationships with other people or organization that could influence this study. 


\section{References}

1. Scherder E, Eggermont L, Swaab D, van Heuvelen M, Kamsma Y, de Greef $M$, van Wijck R, Mulder T: Gait in ageing and associated dementias: its relationship with cognition. Neurosci Biobehav Rev 2007, 31:485-497.

2. Gabell A, Nayak US: The effect of age on variability in gait. J Gerontol 1984, 39:662-666.

3. Beauchet O, Allali G, Annweiler C, Bridenbaugh S, Assal F, Kressig RW, Herrmann FR: Gait variability among healthy adults: low and high strideto-stride variability are both a reflection of gait stability. Gerontology 2009, 55:702-706.

4. Watson NL, Rosano C, Boudreau RM, Simonsick EM, Ferrucci L, SuttonTyrrell K, Hardy SE, Atkinson HH, Yaffe K, Satterfield S, Harris TB, Newman $A B$, Health $A B C$ Study: Executive function, memory, and gait speed decline in well-functioning older adults. J Gerontol A Biol Sci Med Sci 2010, 65:1093-1100.

5. Hausdorff JM, Yogev G, Springer S, Simon ES, Giladi N: Walking is more like catching than tapping: gait in the elderly as a complex cognitive task. Exp Brain Res 2005, 164:541-548.

6. Allali G, Dubois B, Assal F, Lallart E, de Souza LC, Bertoux M, Annweiler C, Herrmann FR, Levy R, Beauchet O: Frontotemporal dementia: pathology of gait? Mov Disord 2010, 25:723-729.

7. Yogev-Seligmann G, Hausdorff JM, Giladi N: The role of executive function and attention in gait. Mov Disord 2008, 23:329-42.

8. Miyake A, Friedman NP, Emerson MJ, Witzki AH, Howerter A, Wager TD: The unity and diversity of executive functions and their contributions to complex "Frontal Lobe" tasks: a latent variable analysis. Cogn Psychol 2000, 41:49-100.

9. Wechsler D: WMS-R:Wechsler Memory Scale - revised manual. San Antonio, TX: The Psychological Corporation, Harcourt Brace Jovanovich; 1987.

10. Brown EC, Casey A, Fisch Rl, Neuringer C: Trial making test as a screening device for the detection of brain damage. J Consult Psychol 1958, 22:469-74.

11. Stroop JR: Studies of interference in serial verbal reactions. J Exp Psychol 1935, 18:643-662.

12. Sakong J, Kang PS, Kim CY, Hwang TY, Jeon MJ, Park SY, Lee SJ, Won KC, Lee SB, Chung $\mathrm{JH}$ : Evaluation of reliability of traditional and computerized neurobehavioral tests. Neurotoxicology 2007, 28:235-239.

13. Waters GS, Caplan D: The reliability and stability of verbal working memory measures. Behav Res Methods Instrum Comput 2003, 35:550-564.

14. Beauchet O, Herrmann FR, Grandjean R, Dubost V, Allali G: Concurrent validity of SMTEC footswitches system for the measurement of temporal gait parameters. Gait Posture 2008, 27:156-159.

15. Kressig RW, Beauchet O, European GAITRite Network Group: Guidelines for clinical applications of spatio-temporal gait analysis in older adults. Aging Clin Exp Res 2006, 18:174-176.

16. Beauchet O, Freiberger E, Annweiler C, Kressig RW, Herrmann FR, Allali G: Test-retest reliability of stride time variability while dual tasking in healthy and demented adults with frontotemporal degeneration. $J$ Neuroeng Rehabil 2011, 8:37.

17. Ble A, Volpato S, Zuliani G, Guralnik JM, Bandinelli S, Lauretani F, Bartali B, Maraldi C, Fellin R, Ferrucci L: Executive function correlates with walking speed in older persons: the InCHIANTI study. J Am Geriatr Soc 2005, 53:410-415.

18. Murray ME, Senjem ML, Petersen RC, Hollman JH, Preboske GM, Weigand SD, Knopman DS, Ferman TJ, Dickson DW, Jack CR Jr: Functional impact of white matter hyperintensities in cognitively normal elderly subjects. Arch Neurol 2010, 67:1379-1385.

doi:10.1186/1743-0003-9-12

Cite this article as: Beauchet et al:: Gait control: a specific subdomain of executive function? Journal of NeuroEngineering and Rehabilitation 2012 9:12

\section{Submit your next manuscript to BioMed Central and take full advantage of:}

- Convenient online submission

- Thorough peer review

- No space constraints or color figure charges

- Immediate publication on acceptance

- Inclusion in PubMed, CAS, Scopus and Google Scholar

- Research which is freely available for redistribution

Submit your manuscript at www.biomedcentral.com/submit
Ciomed Central 